\title{
Analog Circuit Soft Fault Diagnosis Based on Chaotic Neural Network
}

\author{
Meirong Liu ${ }^{1,2, *}$, Li Zeng ${ }^{2}$, Liwei Zhang ${ }^{2,3}$ and Yigang $\mathrm{He}^{4}$ \\ ${ }^{1}$ Electric Engineering Postdoctoral Center, Hefei University of Technology, Hefei 230009, China \\ ${ }^{2}$ College of Physics and Information Science, Hunan Normal University, Changsha 410081, China \\ ${ }^{3}$ China Unicom Chenzhou Branch, Chenzhou 423000, China \\ ${ }^{4}$ College of Electrical and Automation Engineering, Hefei University of Technology, Hefei, 230009 \\ ${ }^{*}$ Corresponding author
}

\begin{abstract}
In order to solve the fault feature redundancy problem in analog circuit fault diagnosis, a method of fault diagnosis is presented in this paper. This approach using the method of wavelet decomposition and fuzzy clustering on the fault signals to obtain test matrix; then inputted the test matrix into the neural network for fault diagnosis. The approach are combined the chaotic motion's ergodic, randomness and sensitive of the initial value to optimize the neural network for making the network have a better learning ability and have a more faster convergence speed to improve the efficiency of fault diagnosis. The simulation results verify the effectiveness of this approach.
\end{abstract}

Keywords-analog circuit; soft fault diagnosis; chaotic; neural network; fuzzy clustering

\section{INTRODUCTION}

The analog circuit fault diagnosis was a difficult problem due to its non-linear, component of tolerance and diversity of fault. Because of the ability of leaning, classification, reasoning, the stronger robustness and adapting ability that the neural network was widely used in recent years. If the input samples of neural network was complex that the neural network was easy to be trapped in local minimum and were converged slowly. As a phenomenon found in non-linear dynamic system chaos had the characteristics of random-like behavior and ergodicity. It could overcome the defects of neural network such as easy to be trapped in local minimum and so on by using these characteristic of chaotic neural network. A fault diagnosis method for analog circuits which combine chaos characteristics and neural network was proposed in the paper. The fault feature extracted by wavelet decomposition and fuzzy clustering was inputted to the network to train the neural network then using the trained neural network to fault diagnosis followed.

\section{FaILURE FEATURE EXTRACTION BY WAVELET DECOMPOSITION AND FUZZY CLUSTERING}

If using the fault information directly obtained from circuit which was contained a large amount of redundant information and noise would cause larger computation and affect the diagnosis result. Wavelet decomposition was a common method of signal processing and widely used in field of signal processing. It could decompose a time signal into an approximation sequence and a detail sequence for further analysis and processing. Figure I was the fault signal wave which was reconstructed by coefficient of the wavelet and it could reflect fault information on better. So the coefficient of the wavelet could be used as the input of neural network as the fault feature.

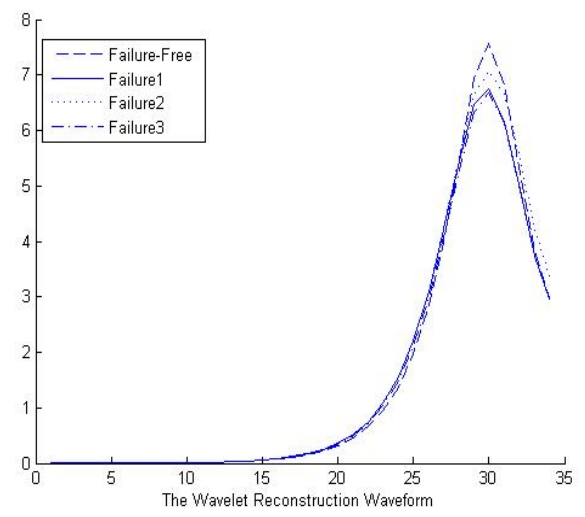

FIGURE I. THE WAVELET RECONSTRUCTION WAVEFORM

High-level of wavelet decomposition would lost fault information and low-level of wavelet could save the fault information but it would cause large dimension of fault information to increase the input of neural network. Fuzzy cluster was using mathematical method to studies the relationship of the sample and could classify the fault feature for reducing the dimension of the fault data with keeping the original of the fault data information complete on better[5-6].

Set the original data matrix as shown in (1):

$$
M=\left[\begin{array}{cccc}
x_{11} & x_{12} & \ldots & x_{1 m} \\
x_{21} & x_{22} & \ldots & x_{2 m} \\
\ldots & \ldots & \ldots & \\
x_{n 1} & x_{n 2} & \ldots & x_{n m}
\end{array}\right]
$$

$$
x_{i j}(i=1, \cdots n ; j=1, \cdots, m) \text { represent the first } j
$$
index of the first $i$ classification. Firstly the collected data were standardized and compressed into the interval [0 1 1].Then the fuzzy similarity matrix $R=\left[\left(r_{i j}\right)_{m \times n}\right]$ is established by using method of fuzzy cluster.

$$
r_{i j}=1-c d\left(x_{i}, x_{j}\right)
$$




$$
d\left(x_{i}, x_{j}\right)=M_{d}=\sqrt{\left(x_{i}-x_{j}\right)^{T} D^{-1}\left(x_{i}-x_{j}\right)}
$$

$C$ was referred to as the parameter that properly chosen to make $0<r_{i j}<1$ in the formula, $M_{d}$ represent Markov distance , $d$ stand for distance. Using the transitive closure $t(R)=R^{*}$ to transform the similarity matrix into fuzzy equivalence matrix $R^{*}$.

$$
R^{*}=\left[\begin{array}{ccc}
a_{11}^{\lambda} & \ldots & \ldots \\
\ldots & \ldots & \ldots \\
a_{i 1}^{\lambda} & \ldots & a_{i j}^{\lambda}
\end{array}\right]
$$

The cutting matrix of $M$ could divide matrix $M$ and it would got several different clusters when $\lambda$ decrease from 1 to 0 . If $a_{i j}>\lambda$ that $a_{i j}=1$ and if not then $a_{i j}=0$. So for different parameter $\lambda$, there were different classification which form a dynamic clustering figure. According to actual demand to tuning the parameter $\lambda$ in the dynamic clustering figure to get cutting matrix and got classification $\left\{x_{i 1} \ldots\right\}, \ldots$ 、 $\left\{X_{m 1} ..\right\}$..As that these data were in the same classification represent they had high degree of similarity and could be merged into the one. With this approach you could extract the appropriate data in large fault information and without reducing too much original fault information.

\section{Chaotic Neural Network}

\section{A. Chaotic Algorithm}

Chaos was a periodic phenomenon in deterministic non-linear dynamic system. Using pseudorandomness and ergodicity of chaotic variables to improve the global search function of neural network. Chaotic variables were generated by chaotic equation. Giving a initial value to the chaotic equation, it would produces a set of random sequence with ergodicity and pseudo- randomness through iteration. Logistic equation was generally selected to generate chaotic sequence and it was a typical one-dimensional chaotic mapping as shown in (5):

$$
x_{n+1}=f\left(a, x_{n}\right)=a x_{n}\left(1-x_{n}\right)
$$

As $a>1+\sqrt{8}$ the system enter chaos state via a period-doubling route and the generated sequence $\left\{x_{n}\right\}$ was chaotic sequence which was ergodic in all state in square $[0,1]$ without repetition. Figure II show that when value of $u$ over critical value that the value of $x$ start bifurcation and random fluctuation in square $[0,1]$ and the system was in a state of chaos.

According to the characteristic of logistic mapping the initial information was gradually lost as the increase of iteration times, so this would leads to long-term unpredictability of chaos.

\section{B. Chaotic Neural Network Constructed by Chaotic Neuron}

There were "internal method" and "external method" to use the method of chaotic optimization neural network. The "internal method" was change parameters of neurons and made the network show chaotic dynamical behavior. This paper proposed an "internal method" based on chaos and neural network in using chaotic algorithm construct chaotic neuron. The figure III was the structure of chaotic neuron.

The chaotic neuron different form normal neuron was that the sum area of normal neuron was divided into three parts: A, B, C. The core of study in neural network was the adjustment of weights. The part A was chaotic part and its value were neither derived from iteration nor affected by external input and made the weights of neuron in state of chaos. Part b was the sum of other neurons to this neuron and it represent the influence of other neurons in this neuron. Part $\mathrm{C}$ was the sum of part A and part B. figure IV was BP network based on chaotic neuron. It changes the hidden layer neurons into chaotic neurons. Because that part of the neuron was chaotic sequences which generated by logistic mapping iterative so the hidden layer would be in chaotic and it equivalent to introduce a chaotic noise to make hidden layer in dynamic.

As neural network input/output relationship was shown in (6) and (7).

$$
y_{k}=f\left(\sum_{i=1}^{m}\left(x_{i} w_{i}+w_{\text {chaos }}\right)\right)
$$

$$
y_{i}=f\left(\sum_{j=1}^{n} y_{k}\right)
$$

In (6) and (7), $m$ was the number of neurons in hidden layer, $n$ stand for the number of neurons in output layer, $w_{\text {chaos }}$ stand for chaotic weights, $y_{k}$ was the input of input payer and $y_{i}$ was the output. Finally we could use the structured chaotic neural network for fault diagnosis.

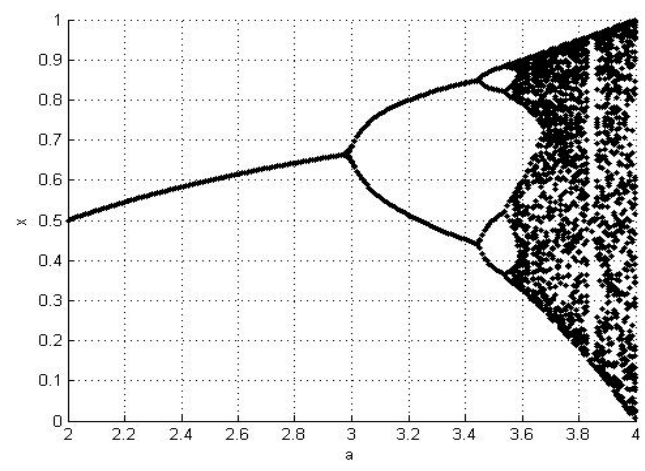

FIGURE II. LOGISTIC BIFURCATION DIAGRAM 


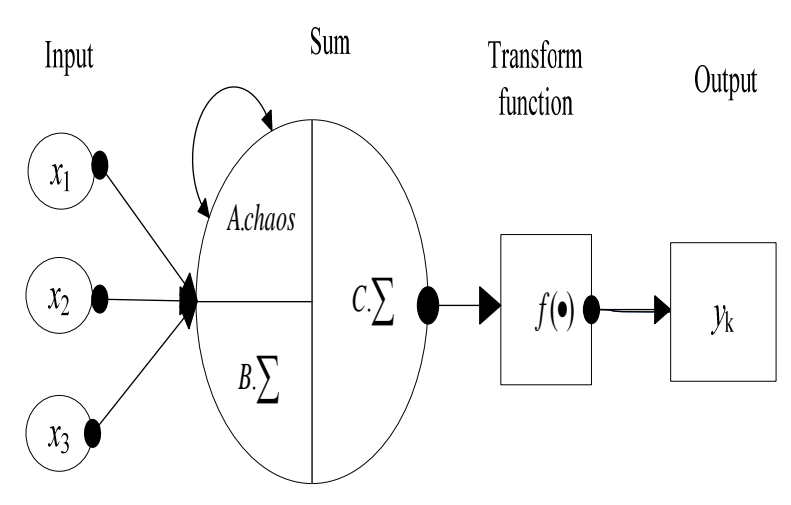

FIGURE III. CHAOTIC NEURON

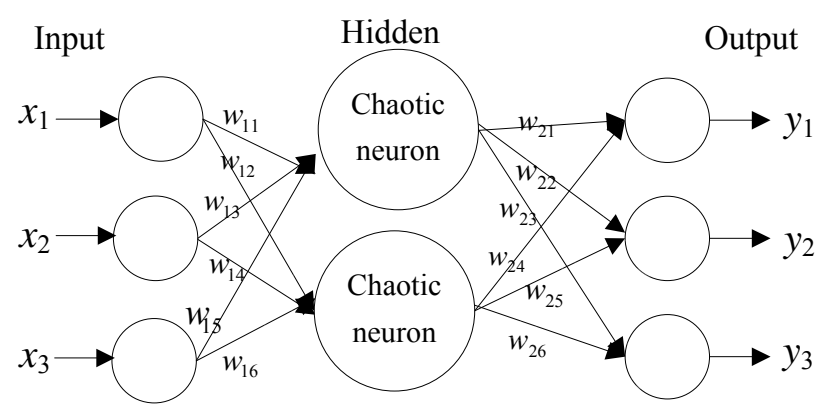

FIGURE IV. DIAGRAM OF CHAOTIC NEURAL NETWORK

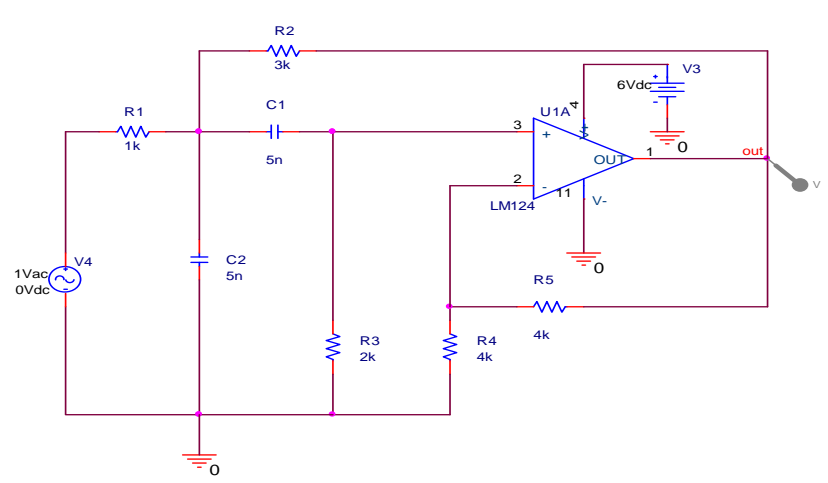

FIGURE V. SALL-KEY BANDPASS FILTER

\section{SimUlation AND ANALYSIS}

In order to verify the method introduced in this article it conduct simulation by using MATLAB software. Taking Sall-key band-pass filter shown in figure $\mathrm{V}$ as example to introduce the analog circuit fault diagnosis in this article. The values of circuit elements in circuit was shown in figure $\mathrm{V}$ and the tolerance of resistor and capacitor was 5\%. The fault condition could be the response of circuit that when one component above or below normal value and other component was in norm. $\uparrow$ represent the component deviates from its normal value from $+50 \%$, $\downarrow$ represent the component deviates from its normal value from $-50 \%$. So there were 9 failure mode listed in the table I.
The AC $2 \mathrm{v}$ was sent to the circuit and 50 times Monte Carlo analysis was conducted, then data sampled at the output. The wavelet analysis and data processing was made based on the sampled data and extract the reconstructed signal after the process of normalization. Finally the data were further analyzed using fuzzy cluster analysis to got 450 groups of fault samples. Part of the fault samples as shown in table II. 360 groups of samples in the 450 as training data and the remained sample to do as test data. Part of the test samples as shown in table III. Structuring a normal neural network and a chaotic neural network which the constriction of neural network shown in table IV.

TABLE I. FAILURE MODE

\begin{tabular}{|c|c|c|c|}
\hline $\begin{array}{c}\text { Fault } \\
\text { number }\end{array}$ & $\begin{array}{c}\text { Fault } \\
\text { type }\end{array}$ & $\begin{array}{c}\text { Fault } \\
\text { number }\end{array}$ & $\begin{array}{c}\text { Fault } \\
\text { type }\end{array}$ \\
\hline F0 & Normal & F5 & $\mathrm{R} 2 \uparrow$ \\
\hline F1 & $\mathrm{C} 1 \uparrow$ & $\mathrm{F} 6$ & $\mathrm{R} 2 \downarrow$ \\
\hline F2 & $\mathrm{C} 1 \downarrow$ & $\mathrm{F} 7$ & $\mathrm{R} 4 \uparrow$ \\
\hline F3 & $\mathrm{C} 2 \uparrow$ & $\mathrm{F} 8$ & $\mathrm{R} 4 \downarrow$ \\
\hline F4 & $\mathrm{C} 2 \downarrow$ & & \\
\hline
\end{tabular}

The neural network was trained through the input fault samples. The convergence of training were shown in figure VI and figure VII.

The statistical result of training were shown in table 4 . According to table 4 the convergence steps of normal neural network was more than 1000 but the convergence steps of chaotic neural network was 324 . Using chaotic neural network improve the speed of fault diagnosis.

In order to precision of diagnosis of chaotic neural network the test samples were inputted in the neural network for testing and the result were shown in table $\mathrm{V}$.

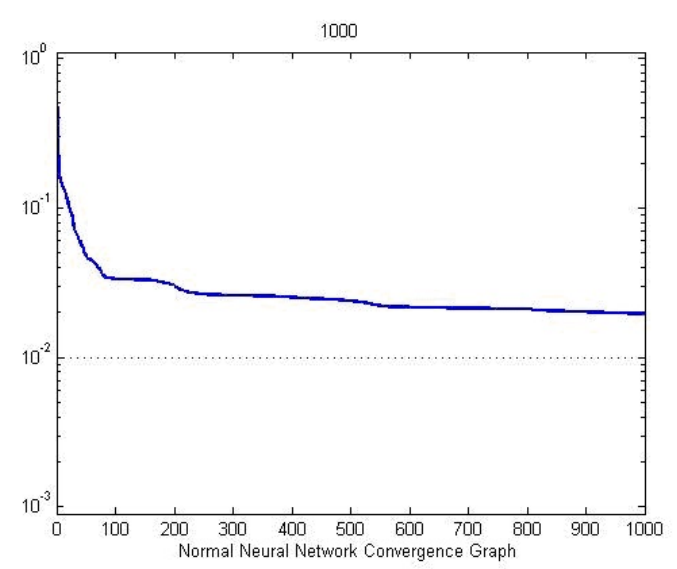

FIGURE VI. NORMAL NEURAL NETWORK CONVERGENCE GRAPH 
TABLE II. PART OF THE FAULT SAMPLES

\begin{tabular}{|c|c|c|c|c|c|c|c|c|c|}
\hline & $\mathbf{1}$ & $\mathbf{2}$ & $\mathbf{3}$ & $\mathbf{4}$ & $\mathbf{5}$ & $\mathbf{6}$ & $\mathbf{7}$ & $\mathbf{8}$ & $\mathbf{O u t p u t}$ \\
\hline F0 & 0.0256 & 0.0536 & 0.2313 & 0.3296 & 0.6309 & 0.9594 & 0.7455 & 0.4405 & 0000 \\
\hline F1 & 0.0294 & 0.0615 & 0.2638 & 0.3732 & 0.6871 & 0.9756 & 0.7652 & 0.4661 & 0001 \\
\hline F2 & 0.0176 & 0.0368 & 0.1605 & 0.2317 & 0.4784 & 0.8907 & 0.6823 & 0.3684 & 0010 \\
\hline F3 & 0.0224 & 0.0469 & 0.2028 & 0.2897 & 0.5628 & 0.9059 & 0.8557 & 0.5467 & 0011 \\
\hline F4 & 0.0293 & 0.0614 & 0.2647 & 0.3751 & 0.7032 & 1.0000 & 0.6735 & 0.3839 & 0100 \\
\hline F5 & 0.0342 & 0.0715 & 0.3046 & 0.4281 & 0.7607 & 1.0000 & 0.6844 & 0.3998 & 0101 \\
\hline F6 & 0.0168 & 0.0352 & 0.1530 & 0.2201 & 0.4452 & 0.8070 & 0.8895 & 0.5607 & 0111 \\
\hline F7 & 0.0284 & 0.0594 & 0.2561 & 0.3645 & 0.6904 & 1.0000 & 0.6728 & 0.3815 & 1000 \\
\hline F8 & 0.0232 & 0.0485 & 0.2093 & 0.2987 & 0.5758 & 0.9117 & 0.8594 & 0.5546 & 1010 \\
\hline
\end{tabular}

TABLE III. PART OF THE TEST SAMPLE

\begin{tabular}{|c|c|c|c|c|c|c|c|c|c|}
\hline $\mathbf{1}$ & $\mathbf{2}$ & $\mathbf{3}$ & $\mathbf{4}$ & $\mathbf{5}$ & $\mathbf{6}$ & $\mathbf{7}$ & $\mathbf{8}$ & $\begin{array}{c}\text { Expected } \\
\text { Output }\end{array}$ & $\begin{array}{c}\text { Fault } \\
\text { number }\end{array}$ \\
\hline 0.0255 & 0.0533 & 0.2300 & 0.3278 & 0.6284 & 0.9584 & 0.7451 & 0.4399 & 0000 & F0 \\
\hline 0.0267 & 0.0559 & 0.2407 & 0.3423 & 0.6479 & 0.9645 & 0.7522 & 0.4488 & 0001 & F1 \\
\hline 0.0239 & 0.0500 & 0.2165 & 0.3096 & 0.6026 & 0.9496 & 0.7355 & 0.428 & 0010 & F2 \\
\hline 0.0302 & 0.0631 & 0.2713 & 0.3849 & 0.7171 & 1.0000 & 0.6499 & 0.3676 & 0011 & F3 \\
\hline 0.0208 & 0.0436 & 0.1885 & 0.2696 & 0.5268 & 0.8684 & 0.9106 & 0.6171 & 0100 & F4 \\
\hline 0.0263 & 0.0551 & 0.2376 & 0.3384 & 0.6444 & 0.9669 & 0.7382 & 0.4352 & 0101 & F5 \\
\hline 0.0246 & 0.0514 & 0.2221 & 0.3169 & 0.6116 & 0.9495 & 0.7533 & 0.4458 & 0111 & F6 \\
\hline 0.0241 & 0.0505 & 0.2181 & 0.3111 & 0.5986 & 0.9333 & 0.7968 & 0.4879 & 1000 & F7 \\
\hline 0.027 & 0.0565 & 0.2439 & 0.3475 & 0.6627 & 0.9851 & 0.7035 & 0.4047 & 1010 & F8 \\
\hline
\end{tabular}

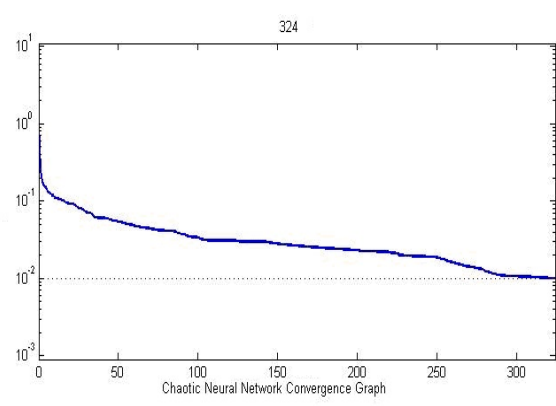

FIGURE VII. CHAOTIC NEURAL NETWORK CONVERGENCE GRAPH

TABLE IV. THE NETWORK STRUCTURE AND RESULT OF TRAINING

\begin{tabular}{|c|c|c|c|}
\hline & $\begin{array}{c}\text { Neural network } \\
\text { structure }\end{array}$ & $\begin{array}{c}\text { Cut-off } \\
\text { error }\end{array}$ & $\begin{array}{c}\text { Iteration } \\
\text { steps }\end{array}$ \\
\hline $\begin{array}{c}\text { Normal neural } \\
\text { network }\end{array}$ & $8-12-4$ & 0.01 & $>1000$ \\
\hline $\begin{array}{c}\text { Chaotic neural } \\
\text { network }\end{array}$ & $8-12-4$ & 0.01 & 324 \\
\hline
\end{tabular}

TABLE V. THE TEST RESULTS

\begin{tabular}{|c|c|c|}
\hline Test sample number & Confirmed number & Success rate \\
\hline 90 & 85 & $94.4 \%$ \\
\hline
\end{tabular}

\section{CONCLUSiOn}

A method of analog circuit fault diagnosis based on chaotic neural network was presented in this paper. This approach use the method of wavelet decomposition and fuzzy clustering on the response of each mode about circuit to reduce the dimension of input. Improve global searching performance of neural network by using the chaotic characteristic of chaotic neuron and had a faster convergence speed to solve the problem which convergence was too slow and avoid minimum value in the normal neural network. The simulation results show that the chaotic neural network had faster rate of convergence and a better diagnosis effect than normal neural network.

\section{ACKNOWLEDGMENT}

This work was supported by National Natural Science Foundation of China under Grant No.60876022.

\section{REFERENCES}

[1] Jin Y, Chen G Y, LIU H. Fault diagnosis of analog circuit based on wavelet neural network[J]. 2007, 28(9): 1600-1604

[2] He Y G, Tan Y-H. A neural-network approach for fault diagnosis of large-scale analog circuits[A]. Proc IEEE ISCAS[C].USA, 2002.153-156

[3] H.G.S. Deterministic Chaos- An Introduction[M].Cheng du; Sichuan education publishing house, 2010 
[4] Liu M R, He Y G. Location for Fault Diagnosis of Analog Circuits Based on GA, Wavelet and $\mathrm{NN}[\mathrm{J}]$. Chinese Journal of Scientific Instrument, Journal of Hunan University(Natural Sciences), 2009, 36(3): 40-44

[5] Xiao Y Q, He Y G. A Fault Diagnosis Approach for Analog Circuits Based on Wavelet Fractal Analysis and Ridgelet Network[J]. TRANSACTIONS OF CHINA ELECTROTECHNICAL SOCIETY, 2011, 26(11): 105-114

[6] Huang L, Hou J J, Liu Y. Fault analysis of hierarchical cluster and fault diagnosis of Mahalanobis distance in analog circuit[J]. JOURNAL OF ELECTRONIC MEASUREMENT AND INSTRUMENT, 2010, 2(7): 610-615

[7] Wang T Y, Cheng W D, Li J Y. Optimal evaluation of feature extraction method based on three types of measure values[J].Chinese Journal of Scientific Instrument, 2010, 31(4): 898-903

[8] Qu F H, Cui G C, Li Y F, Hu Y T. Fuzzy clustering algorithm and its application[M], Beijing: National defence industry press,2011

[9] Song G M, Wang H J, Jiang S Y, Liu H. Analog circuit fault diagnosis approach using clustering and hierarchical decision SVM[J]. Chinese Journal of Scientific Instrument, 2010, 31(5): 998-1104

[10] Han H, Wang H J, Long B, Liu Z. Method for analog circuit fault diagnosis based on improved Mahalanobis distance[J]. Control and Decision, 2013, 28(11): 1714-1717

[11] Wang Y N, Yu Q M, Yuan X F. Progress of Chaotic Neural Networks and Their Applications[J]. Control and Decision, 2006, 21(2):121-128

[12] Xu Y M, Sun M. Chaotic neural network and its application[M]. Harbin; Heilongjiang university press, 2012

[13] Aminian M, Aminian F. A modular fault-diagnostic system for analog electronic circuits using neural networks with wavelet transform as a preprocessor[J]. IEEE Transactions on Instrumentation and Measurement, 2007, 56(9):1546-1554

[14] Qin G X. Application of Chaos in BP Algorithm[J]. Computer simulation, 2011, 28(6):215-218

[15] Liu M R, He Y G, Zhao X M. Chaos Method for Analog Circuit Fault Diagnosis[J]. Journal of Natural Science of Hunan Normal University, 2012, 35(3):34-39 